\title{
Merlin, a regulator of Hippo signaling, regulates Wnt/ $\beta$-catenin signaling
}

\author{
Soyoung Kim E Eek-hoon Jho ${ }^{*}$ \\ Department of Life Science, University of Seoul, Seoul 02504, Korea
}

\begin{abstract}
Merlin, encoded by the NF2 gene, is a tumor suppressor that exerts its function via inhibiting mitogenic receptors at the plasma membrane. Although multiple mutations in Merlin have been identified in Neurofibromatosis type II (NF2) disease, its molecular mechanism is not fully understood. Here, we show that Merlin interacts with LRP6 and inhibits LRP6 phosphorylation, a critical step for the initiation of Wnt signaling. We found that treatment of Wnt3a caused phosphorylation of Merlin by PAK1, leading to detachment of Merlin from LRP6 and allowing the initiation of Wnt/ $\beta$-catenin signaling. A higher level of $\beta$-catenin was found in tissues from NF2 patients. Enhanced proliferation and migration caused by knockdown of Merlin in glioblastoma cells were inhibited by suppression of $\beta$-catenin. Conclusively, these results suggest that sustained $W n t / \beta$-catenin signaling activity induced by abrogation of Merlin-mediated inhibition of LRP6 phosphorylation might be a cause of NF2 disease. [BMB Reports 2016; 49(7): 357-358]
\end{abstract}

Merlin, a tumor-suppressive protein encoded by NF2 gene, is mainly localized to the plasma membrane. It interacts with a number of membrane proteins. Merlin exerts its tumor suppressive effects on multiple mitogenic signaling pathways via modulating interaction with growth factor receptors. Furthermore, Merlin activates the Hippo pathway and turns off YAP/TAZ mediated expression of genes involved in proliferation and anti-apoptosis (Pan et al. 2010. Dev cell 19,

*Corresponding author. E-mail: ej70@uos.ac.kr

http://dx.doi.org/10.5483/BMBRep.2016.49.7.104

Received 24 June 2016

Keywords: LRP6, Merlin, NF2, PAK, Wnt/ $\beta$-catenin

Abbreviations: dnPAK1, dominant-negative form of PAK1; NF2, Neurofibromatosis type II; PAK, p21 activated kinase; PIP 2 , phosphatidylinositol 4,5 bisphosphate; Wnt-CM, Wnt3a-conditioned media

Perspective to: Kim et al. (2016), Merlin inhibits Wnt/ $\beta$-catenin signaling by blocking LRP6 phosphorylation, Cell Death and Differentiation, 10 June 2016; doi:10.1038/cdd.2016.54
491-505). Recent studies have suggested that Merlin can inhibit $\mathrm{Wnt} / \beta$-catenin signaling through inhibiting phosphorylation of $\beta$-catenin, thus blocking the translocation of $\beta$-catenin from membrane to nucleus by inhibiting dissociation of $\beta$-catenin from adherens junction (Bosco et al. 2010. Oncogene 29, 2540-9; Zhou et al. 2011. Neoplasia 13, 1101-12). Although the functional relationship between growth factor receptor and Merlin has been extensively studied, the role of Merlin in $\mathrm{Wnt} / \beta$-catenin signaling at receptor level has not been demonstrated. Our study showed that active Merlin, a de-phosphorylated form of Merlin on Ser 518, is a binding partner of Wnt co-receptor LRP6. Interaction between Merlin and LRP6 was inhibited by incubation with Wnt-conditioned media (Wnt-CM). We also showed that knockdown of Merlin enhanced Wnt reporter activity whereas overexpression of Merlin inhibited Wnt reporter activity. In addition, secondary axis induced by the injection of a constitutive active form of LRP6 into ventral side of Xenopus embryos was significantly reduced by the co-injection of Merlin. Overall, these data suggest that Merlin can inhibit $\mathrm{Wnt} / \beta$-catenin signaling via interaction with LRP6.

Except for Merlin, mutations in components of Hippo pathway are uncommon in human cancer. It seems that the increase in the level of YAP/TAZ alone may not be enough to cause human cancer. Additional mutations are required. Our results showed that not the level of YAP, a terminal regulator of Hippo pathway, but $\beta$-catenin was significantly increased in all schwannomas isolated from NF2 patients compared to that in normal adjacent tissues. We found that enhanced glioblastoma cell growth and migration induced by the suppression of Merlin were restored by depletion of $\beta$-catenin. Thus, our data illustrate that NF2 disease with Merlin mutations might be caused mainly by the activation of $\mathrm{Wnt} / \beta$-catenin signaling in addition to enhanced YAP/TAZ signaling.

LRP6 phosphorylation is critical for the activation of $W n t / \beta$-catenin signaling. We showed that Merlin interacted with LRP6 and suppressed $\mathrm{Wnt} / \beta$-catenin signaling upstream of $\beta$-catenin by blocking phosphorylation of LRP6. We have previously demonstrated that formation of $\mathrm{PIP}_{2}$ by Arf1, a small G protein, is necessary for the phosphorylation of LRP6 (Kim et al. 2013. Oncogene 32, 3390-3396). Similarly, our current finding revealed that $\mathrm{PIP}_{2}$ could be used as a docking 


\section{a. Wnt OFF}

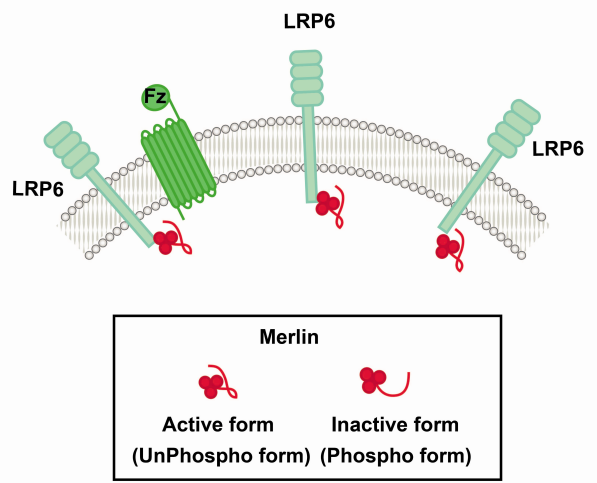

\section{b. Wnt ON}

LRP6

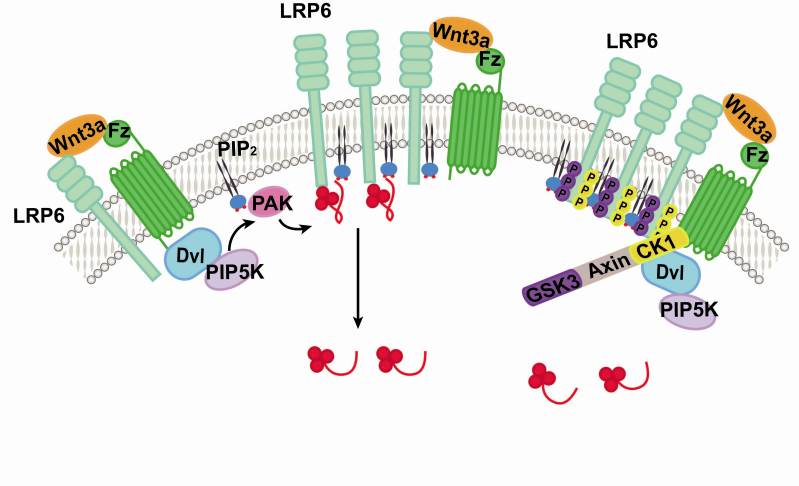

Fig. 1. Schematic model. (A) Wnt-off condition. In the absence of Wnt or below a certain threshold of Wnt, active merlin bind to LRP6 and inhibits Wnt/ $\beta$-catenin signaling. (B) In the presence of Wnt, the level of PIP2 is increased, which could be used as a docking site for PAK1. Active PAK1 phosphorylates Merlin on Ser518, resulting in detachment of Merlin from LRP6 and allowing activation of LRP6 for the initiation of $\mathrm{Wnt} / \beta$-catenin signaling.

site for PAK1 (p21 activated kinase). In the presence of Wnt3a-CM, activated PAK1 (p21 activated kinase) can bind to $\mathrm{PIP}_{2}$ and phosphorylate Merlin on Ser 518, thus inducing the detachment of Merlin from LRP6 and allowing phosphorylation of LRP6 for the initiation of $\mathrm{Wnt} / \beta$-catenin signaling (Fig. 1).

Overall, our current study reveals that active Merlin can bind to LRP6 and inhibit the initiation of $\mathrm{Wnt} / \beta$-catenin signaling, which may block non-specific activation of $\mathrm{Wnt} / \beta$ catenin signaling until the level of Wnt ligand is above certain threshold. Although we showed that the detachment of Merlin from LRP6 allowed the activation of $\mathrm{Wnt} / \beta$-catenin signaling, the role of detached Merlin from LRP6 in the regulation of Hippo signaling needs to be further studied in the future. Specific regulation of $\mathrm{Wnt} / \beta$-catenin signaling with minimal influence of other signaling pathways is extremely valuable for the purpose of developing therapeutics. Small molecules that may control the interaction between Merlin and LRP6 could be very specific modulators of $\mathrm{Wnt} / \beta$-catenin signaling.

\section{ACKNOWLEDGEMENTS}

This work was supported by the 2015 Research Fund of the University of Seoul, Korea. 